\title{
Avaliação do nível de osteartrose de joelhos, risco de quedas e funcionalidade em
}

\author{
idosos \\ Assessment of the risk of falls and functional capacity in the elderly with knee osteoarthritis \\ Evaluación del nivel de artrosis de rodilla, riesgo de caídas y funcionalidad en ancianos
}

Recebido: 30/09/2021 | Revisado: 09/10/2021 | Aceito: 15/10/2021 | Publicado: 17/10/2021

\author{
Aline Micheline da Silva Melo \\ ORCID: https://orcid.org/0000-0002-3269-062X \\ Universidade Católica de Pernambuco, Brasil \\ E-mail: alinemicheline@ hotmail.com \\ Juliana Malveira Pereira \\ ORCID: https://orcid.org/0000-0002-2961-858X \\ Universidade Católica de Pernambuco, Brasil \\ E-mail: julianamalveira20@gmail.com \\ Keylla Silva Alves de Melo \\ ORCID: https://orcid.org/0000-0001-6019-1433 \\ Universidade Católica de Pernambuco, Brasil \\ E-mail: keyllasilva-melo@hotmail.com \\ Valéria Conceição Passos de Carvalho \\ ORCID: https://orcid.org/0000-0001-8314-9000 \\ Universidade Católica de Pernambuco, Brasil \\ E-mail: valeriapassos@gmail.com \\ Marina de Lima Neves Barros \\ ORCID: https://orcid.org/0000-0002-3544-0538 \\ Universidade Católica de Pernambuco, Brasil \\ E-mail: marinalnbarros@gmail.com \\ Érica Patrícia Borba Lira Uchôa \\ ORCID: https://orcid.org/0000-0003-4099-1876 \\ Universidade Católica de Pernambuco, Brasil \\ E-mail: ericaluchoa@gmail.com
}

\begin{abstract}
Resumo
Introdução: A osteoartrose é uma patologia crônico-degenerativa, com alta prevalência entre idosos, que acarreta dor, rigidez articular e diminuição da mobilidade, promovendo assim, incapacidade funcional e impacto negativo sobre a qualidade de vida. Objetivo: Avaliar o nível de osteartrose de joelhos, risco de quedas e funcionalidade em idosos. Métodos: Estudo observacional, descritivo, de corte transversal, de caráter quantitativo. A amostra foi constituída de 16 idosos com idade igual ou superior a 60 anos. Utilizou-se o questionário socioclínico para verificar o perfil da população, o WOMAC, para avaliação do nível de osteoartrose de joelhos; as escalas de equilíbrio de Berg e Tinetti para avaliar o equilíbrio e o risco de quedas, e, o Índice de Katz e Escala de Lawton e Brody para avaliação da funcionalidade. Resultados: A população apresentou predomínio de mulheres $(81,3 \%)$, com média de idade de 68,44 $\pm 6,42$ anos e IMC de $28,97 \pm 3,51 \mathrm{Kg} / \mathrm{m}^{2}$. Foi observado que pelo WOMAC, $37.5 \%$ apresentaram pouco comprometimento pela osteoartrose. Na classificação de Berg e na escala de Tinetti, 75,0\% apresentaram bom equilíbrio e não apresentam risco de quedas. E tanto pela escala de Katz e pela classificação de Lawton \& Brody, os idosos foram considerados independentes com o mesmo percentual de 62,5\%. Considerações Finais: O estudo apresentou nível de comprometimento funcional moderado pela osteoartrose, porém com equilíbrio preservado, baixo risco a quedas e com independência funcional preservada tanto para as Atividades Básicas de Vida Diárias (ABVDs) e Atividades Instrumentais de Vida Diárias (AIVDs).
\end{abstract}

Palavras-chave: Funcionalidade; Envelhecimento; Joelho; Osteoartrose.

\begin{abstract}
Introduction: Osteoarthrosis is a chronic-degenerative disease, with high prevalence among the elderly, which causes pain, joint stiffness and decreased mobility, thus promoting functional incapacity and negative impact on quality of life. Objective: to assess the level of osteoarthritis in the knees, risk of falls and functionality in the elderly. Methods: Observational, descriptive, cross-sectional, quantitative study. The sample consisted of 16 elderly people aged over 60 years. Use the socioclinical questionnaire to check the profile of the population, the WOMAC, to assess the level of osteoarthritis in the knees; the Berg and Tinetti balance scales to assess balance and the risk of falls, and the Katz Index and Lawton and Brody Scale to assess functionality. Results: The population showed a predominance of women $(81.3 \%)$, with a mean age of $68.44 \pm 6.42$ years and BMI of $28.97 \pm 3.51 \mathrm{~kg} / \mathrm{m} 2$. It was observed that by WOMAC, $37.5 \%$ showed little impairment by osteoarthrosis. In the Berg classification and Tinetti scale, $75.0 \%$ presented good
\end{abstract}


balance and did not present a risk of falls. And both by the Katz scale and by the Lawton \& Brody classification, the elderly were considered independent with the same percentage of $62.5 \%$. Final Considerations: The study showed a moderate level of functional impairment due to osteoarthrosis, but with preserved balance, low risk of falls and preserved functional independence for both Basic Activities of Daily Living (BADLs) and Instrumental Activities of Daily Living (IADLs).

Keywords: Functioning; Aging; Knee; Osteoarthritis.

\section{Resumen}

Introducción: La osteoartrosis es una enfermedad crónico-degenerativa, con alta prevalencia entre los ancianos, que provoca dolor, rigidez articular y disminución de la movilidad, favoreciendo así la incapacidad funcional e impacto negativo en la calidad de vida. Objetivo: Evaluar el nivel de artrosis de rodillas, riesgo de caídas y funcionalidad en ancianos. Métodos: Estudio observacional, descriptivo, transversal, cuantitativo. La muestra estuvo formada por 16 personas mayores de 60 años. Utilizamos el cuestionario socioclínico para verificar el perfil de la población, el WOMAC, para evaluar el nivel de artrosis en las rodillas; las escalas de equilibrio de Berg y Tinetti para evaluar el equilibrio y el riesgo de caídas, y el índice de Katz y la escala de Lawton y Brody para evaluar la funcionalidad. Resultados: La población mostró un predominio de mujeres (81,3\%), con una edad media de 68,44 \pm 6,42 años e IMC de 28,97 $\pm 3,51 \mathrm{~kg} / \mathrm{m} 2$. Se observó que según WOMAC, el 37,5\% mostraba poco deterioro por osteoartrosis. En la clasificación de Berg y escala de Tinetti, el 75,0\% presentó buen equilibrio y no presentó riesgo de caídas. Y tanto por la escala de Katz como por la clasificación de Lawton \& Brody, los ancianos se consideraron independientes con el mismo porcentaje de $62,5 \%$. Consideraciones finales: El estudio mostró un nivel moderado de deterioro funcional debido a la osteoartrosis, pero con equilibrio conservado, bajo riesgo de caídas e independencia funcional preservada tanto para las Actividades Básicas de la Vida Diaria (BADL) como para las Actividades Instrumentales de la Vida Diaria (IADL).

Palabras clave: Funcionalidad; Envejecimiento; Rodilla; Osteoartrosis.

\section{Introdução}

Envelhecimento é uma etapa da vida, um ciclo natural, que faz com que o corpo apresente mudanças anatômicas e funcionais, com o passar do tempo, ocorrerá dificuldade na execução das atividades da vida diária (AVDs). As alterações funcionais podem prejudicar a funcionalidade global do idoso, vindo a fornecer acometimento dos padrões de postura e de equilíbrio e, posteriormente, exposição a quedas (Ritzel, 2010). Dentro deste processo algumas patologias podem surgir, tais como hipertensão arterial, diabetes e doenças do sistema musculoesquelético, como a osteoartrose (Pinheiro et al., 2019).

A osteoartrose é uma disfunção de caráter degenerativo, natural, que destroem a estrutura cartilaginosa, com alta prevalência entre idosos. Entre os locais de comprometimento da osteoartrose, destaca-se a articulação de joelho, que devido à alta demanda funcional é uma articulação que tem grande sobrecarga e está mais susceptível ao surgimento das alterações, principalmente dor, rigidez articular e diminuição da mobilidade, promovendo assim, incapacidade funcional e impacto negativo sobre a qualidade de vida (Freitas et al., 2002).

A perda da mobilidade é caracterizada pelas restrições das articulações, pois depende dos estímulos sensoriais e da ação muscular. Sendo assim, o estudo da capacidade funcional é um quesito importante para observar o nível de independência funcional, repercutindo no envelhecimento saudável, e é caracterizada quanto à habilidade e independência para realizar determinadas atividades (Marcon et al., 2009).

As alterações promovidas pelo próprio envelhecimento, tais como diminuição das funções neuro-motoras que interferem na mobilidade e flexibilidade do idoso, favorecendo a perda do equilíbrio postural durante as realizações das Atividades de Vida Diária (AVDs) associadas às alterações patológicas da osteoartrose de joelho, podem favorecer ao surgimento das quedas, o qual é um incidente bastante comum; e, que comprometem a qualidade do envelhecer (Santos et al., 2012).

Devido a este crescimento da população idosa, atualmente há uma grande preocupação com os problemas relacionados à saúde, como as alterações promovidas pela osteoartrose; e, também a repercussão das quedas nesta população, provenientes de vários fatores. Diante disso, nota-se a necessidade de avaliar o nível de osteoartrose de joelhos, o risco de quedas e a funcionalidade em idosos. 


\section{Metodologia}

Este projeto foi vinculado a Universidade Católica de Pernambuco a Escola de Saúde e Ciências da Vida e ao curso de Fisioterapia. Foi vinculado ao projeto de pesquisa intitulado "Fisioterapia na saúde de indivíduos idosos: recursos de avaliação e intervenção nas alterações osteomioarticulares”, com o número de CAAE “02868418.4.0000.5206”, já aprovado pelo comitê de ética e pesquisa em seres humanos, com número de parecer: 3.049 .932 e pertencente ao grupo de Fisioterapia baseada em Evidências.

É um estudo do tipo observacional, descritivo, de corte transversal e de caráter quantitativo. As avaliações foram realizadas através de dispositivo eletrônico, celular e por dispositivo de mensagens, Whatsapp®. O período da coleta de dados foi realizado de novembro de 2020 a abril de 2021.

A amostra foi feita por conveniência, por idosos que aceitassem participar do estudo, sendo incluídos na pesquisa idosos de ambos os sexos, com idade superior a 60 anos, com diagnóstico de osteoartrose de joelho e que assinem o termo de consentimento livre esclarecido (TCLE). E foram excluídos, os idosos com comprometimento cognitivo que impedissem o entendimento dos questionários, que apresentam patologias associadas que interferissem na função e na mobilidade como: amputações, sequelas de traumas e presença de deformidades e neurológico, e pacientes com doenças terminais.

A metodologia do presente trabalho foi formada por quatro etapas que se completam. Na primeira etapa, foi realizada uma palestra explicativa através do dispositivo móvel, sobre os objetivos do estudo. Após a sua concordância para participar da pesquisa, foi encaminhado via e-mail ou por anexo no Whatsapp®, do idoso ou de seu familiar, para que seja assinado o TCLE, atendendo dessa forma à Resolução 466/12 do Conselho Nacional de Saúde, garantindo aos participantes a liberdade de retirar o consentimento em qualquer momento durante a pesquisa.

Após a assinatura do termo, foi iniciada a segunda etapa com a coleta de dados, utilizando um questionário para a obtenção do perfil socioclínico, com dados referentes a sexo, idade, peso, altura, estado civil, ocupação, uso de medicamentos, uso de cigarro, entre outros.

$\mathrm{Na}$ terceira etapa foi aplicado o instrumento para avaliação do nível de osteoartrose, o questionário WOMAC, o qual consta a dor, rigidez e disfunção física e motora. A dimensão da dor é avaliada em 5 itens específicos e suas funções. Para a rigidez, possui 2 questões e para a disfunção física e motora dos movimentos de joelhos, 17 itens são utilizados. Desta forma, este instrumento possui ao todo 24 itens que são avaliados através de um escores que varia do 0 ("nenhum sintoma/ nenhuma limitação") até 5 (“dor máxima/ limitação extrema”), apresentando como pontuação mínima 0 pontos e pontuação máxima 120 pontos, e quanto maior a pontuação maior o nível de dor e incapacidade para o indivíduo. Este valor deve ser transformado em uma escala, na qual a 0 corresponde ao melhor estado; de 1 a 25 , pouco comprometimento; de 26 a 50 , comprometimento moderado; de 51 a 75, comprometimento muito intenso e de 76 a 100, pior estado (Salaffi et al., 2005).

Também foram aplicados escalas para avaliar o risco de quedas, a escala de equilíbrio de Berg e Tinetti; e, para avaliação da funcionalidade, o Índice de Katz, Escala de Lawton e Brody.

A Escala de Equilíbrio de Berg consiste em uma avaliação funcional do equilíbrio. Este apresenta 14 itens comuns do dia a dia, as tarefas envolvem o equilíbrio estático e dinâmico tal como, alcançar, girar, transferir-se, permanecer de pé e levantar-se, possuindo cada item uma escala ordinal de 5 alternativas que variam de 0 a 4 pontos em função do desempenho do indivíduo, onde a pontuação máxima é de 56 pontos. A pontuação de 0 a 20 pontos, refere-se a prejuízo do equilíbrio, 21 a 40 pontos, equilíbrio aceitável e de 41 a 56 pontos, bom equilíbrio. Para a sua aplicação são necessários um cronômetro e uma régua (Da Silva et al., 2008).

O Teste de Tinetti consiste em uma avaliação do equilíbrio e das anomalias da marcha. É formado por de 16 itens, das quais 9 são para o equilíbrio do corpo e 7 para a marcha. Ela avalia as características da marcha como a velocidade, a distância do passo, a simetria e o equilíbrio em pé, o girar e as mudanças com os olhos fechados. Para cada item do teste a pontuação 
varia de 0 a 1 ou de 0 a 2. A pontuação total é de 28 pontos, onde 16 são referentes ao equilíbrio e 12 são referidos a marcha. $O$ resultado obtido pode ser feito de: 0-19 pontos: elevado risco de queda, 19-24 pontos: moderado de queda e 24-28 pontos: baixo risco de queda (Da Silva et al., 2008).

O Índice de Katz avalia a capacidade de realizar as Atividades Básicas de Vida Diária (ABVDs) que são: tomar banho, se vestir, ir ao banheiro, fazer transferência da cama para a cadeira e vice-versa, continência esfincteriana e se alimentar. A classificação identifica o grau de dependência do indivíduo na realização de alguma dessas atividades e varia de A a G e Outros, definidos como: A - Independente para todas as atividades; B - Independente para todas as atividades menos uma; C - Independente para todas as atividades menos banho e mais uma adicional; D - Independente para todas as atividades menos banho, se vestir e mais um adicional; E - Independente para todas as atividades menos banho, se vestir, ir ao banheiro e mais um adicional; F - Independente para todas as atividades menos banho, se vestir, ir ao banheiro, transferência e mais um adicional; G - Dependente para todas as atividades; OUTROS) Dependente em pelo menor duas funções, mas que não se classifique na letra C, D, E, F (Lino et al., 2008).

A Escala de Lawton e Brody avalia a capacidade de realizar as Atividades Instrumentais de Vida Diária (AIVDs). O questionário possui 8 itens: usar o telefone, viajar, fazer compras, preparar refeições, arrumar a casa, realizar atividades manuais, usar medicações, gerir finanças. Cada item vai pontuar de um (1) a três (3), onde o (1) representa independência, o (2) representa dependência parcial e o (3) representa dependência. O somatório mínimo dos itens é de 7 pontos (dependência total) e o máximo é de 21 pontos (independência total (Araújo et al., 2008).

Após o preenchimento dos questionários foi elaborada uma planilha do Excel com todas as variáveis do estudo, para realização da análise descritiva, com cálculos de média, desvio padrão, mínimo e máximo. Para o cálculo dos percentuais das variáveis que apresentaram múltiplas respostas foi utilizado o total de casos e por essa razão, para essas variáveis, a soma ultrapassa $100 \%$.

\section{Resultados}

A população em estudo foi composta por 16 idosos que foram avaliados quanto ao nível da osteoartrose, risco de quedas e funcionalidade através dos instrumentos de WOMAC, Katz, Tinetti, Berg e Lawton \& Brody.

A Tabela 1 apresenta a distribuição dos idosos quanto as características sociodemográficas e clínicas. Nesta destaca-se que $81,3 \%(n=13)$ dos idosos são do sexo feminino, 50,0\% $(n=8)$ dos idosos tem idade superior a 65 anos, $62,6 \%(n=10)$ estão solteiros, viúvos ou divorciados e 75,0\% (n=12), são classificados com sobrepeso segundo a classificação do IMC para idosos. Tem-se ainda que $87,5 \%(n=14)$ residem com algum familiar, $81,3 \%(n=13)$ residem com duas ou mais pessoas e $31,3 \%(n=5)$ apresentam patologias. 
Tabela 1 - Distribuição dos idosos quanto às características sociodemográficas e clínicas.

\begin{tabular}{|c|c|c|}
\hline Características sociodemográficas e clínicas & $\mathbf{N}$ & $\%$ \\
\hline \multicolumn{3}{|l|}{ Sexo } \\
\hline Feminino & 13 & 81,3 \\
\hline Masculino & 3 & 18,8 \\
\hline \multicolumn{3}{|l|}{ Faixa etária } \\
\hline Até 65 & 8 & 50,0 \\
\hline$>65$ & 8 & 50,0 \\
\hline \multicolumn{3}{|l|}{ Estado civil } \\
\hline Solteiro & 5 & 31,3 \\
\hline Casado & 6 & 37,5 \\
\hline Viúvo & 4 & 25,0 \\
\hline Divorciado & 1 & 6,3 \\
\hline \multicolumn{3}{|l|}{ IMC } \\
\hline Baixo peso & 1 & 6,3 \\
\hline Eutrófico & 3 & 18,8 \\
\hline Sobrepeso & 12 & 75,0 \\
\hline \multicolumn{3}{|l|}{ Residem com algum familiar } \\
\hline Sim & 14 & 87,5 \\
\hline Não & 2 & 12,5 \\
\hline \multicolumn{3}{|l|}{ Número de pessoas que residem } \\
\hline Até uma & 3 & 18,8 \\
\hline Duas ou + & 13 & 81,3 \\
\hline \multicolumn{3}{|l|}{ Apresenta patologias } \\
\hline Sim & 5 & 31,3 \\
\hline Não & 11 & 68,8 \\
\hline \multicolumn{3}{|l|}{ Principais patologias } \\
\hline Fascite plantar & 1 & 20,0 \\
\hline Um pouco de desequilíbrio devido a três AVC's & 1 & 20,0 \\
\hline Mastectomia mama esquerda & 1 & 20,0 \\
\hline Punho fraturado & 1 & 20,0 \\
\hline Retenção de líquido & 1 & 20,0 \\
\hline Amplitude do braço esquerdo reduzida & 1 & 20,0 \\
\hline
\end{tabular}

Fonte: Autores (2021).

A Tabela 2 apresenta a distribuição dos idosos com osteoartrose de joelhos quanto idade, altura, peso, IMC e número de pessoas com que residem. Nesta verifica-se que a idade foi, em média, de 68,44 $\pm 6,42$ anos, a altura foi de 1,62 $\pm 0,10 \mathrm{~m}, \mathrm{o}$ peso foi de $76,88 \pm 15,02 \mathrm{Kg}$ e o IMC de $28,97 \pm 3,51 \mathrm{Kg} / \mathrm{m}^{2}$. 
Tabela 2 - Distribuição dos idosos quanto idade, altura, peso e IMC.

\begin{tabular}{llllll}
\hline & N & Média & Desvio & Mínimo & Máximo \\
\hline Idade (anos) & 16 & 68,44 & 6,42 & 60,00 & 83,00 \\
Altura (m) & 16 & 1,62 & 0,10 & 1,50 & 1,82 \\
Peso $(\mathrm{Kg})$ & 16 & 76,88 & 15,02 & 56,00 & 110,00 \\
IMC $\left(\mathrm{Kg} / \mathrm{m}^{2}\right)$ & 16 & 28,97 & 3,51 & 21,60 & 35,44 \\
\hline
\end{tabular}

Fonte: Autores (2021).

A tabela 3 apresenta a distribuição dos idosos quanto aos escores de WOMAC, Tinetti, Berg, Katz e Lawton \& Brody. Nesta verifica-se que no WOMAC, o escore de intensidade de dor foi, em média, de 25,00 $\pm 23,52$, o de rigidez foi de 29,69 \pm 26,95 e o de atividade física, foi de 30,15 $\pm 23,69$. Para a escala de Berg observa-se uma média de 46,50 $\pm 11,48$, sendo classificado como bom equilíbrio. Em relação ao teste de Tinetti, verificamos que o escore de equilíbrio teve média de 13,31 \pm 3,24, o de marcha, 7,06 $\pm 3,45$ e o total, 20,38 $\pm 6,33$, sendo caracterizado como moderado risco de quedas. Na escala de Katz foi de $0,56 \pm 0,89$. E para a escala de Lawton \& Brody foi observada uma média de 19,63 $\pm 2,58$, classificando-se como dependência moderada.

Tabela 3 - Distribuição dos idosos quanto aos escores de WOMAC, Berg, Tinetti, Katz e Lawton \& Brody.

\begin{tabular}{llllll}
\hline & N & Média & Mínimo & Máximo & Desvio \\
\hline WOMAC & 16 & 25,00 & 0,00 & 65,00 & 23,52 \\
Intensidade da dor & 16 & 29,69 & 0,00 & 87,50 & 26,95 \\
Rigidez & 16 & 30,15 & 0,00 & 69,12 & 23,69 \\
Atividade física & 16 & 29,04 & 0,00 & 69,79 & 23,34 \\
Total & 16 & 46,50 & 23,00 & 56,00 & 11,48 \\
Escala de Berg & & & & & 3,24 \\
Tinetti & 16 & 13,31 & 6,00 & 16,00 & 3,45 \\
Equilíbrio & 16 & 7,06 & 1,00 & 11,00 & 6,33 \\
Marcha & 16 & 20,38 & 7,00 & 27,00 & 0,89 \\
Total & 16 & 0,56 & 0,00 & 3,00 & 2,58 \\
Escala de Katz & 16 & 19,63 & 11,00 & 21,00 & \\
Escala de Lawton \& Brody & 16 & & & & \\
\hline
\end{tabular}

Fonte: Autores (2021).

A Tabela 4 apresenta a distribuição dos idosos quanto a classificação do WOMAC, da escala de Berg, do risco de queda de Tinetti, da escala de Katz, e da escala de Lawton e Brody. Nesta observa-se que pelo WOMAC, 37.5\% (n=6) apresentaram pouco comprometimento pela osteoartrose, pela classificação de Berg e pela escala de Tinetti, 75,0\% ( $\mathrm{n}=12$ ), apresentam bom equilíbrio e não apresentaram risco de quedas. E tanto pela escala de Katz e pela classificação de Lawton \& Brody, foram considerados independentes com o mesmo percentual de $62,5 \%(n=10)$. 
Tabela 4 - Distribuição dos idosos quanto à classificação pelo de WOMAC e das escalas de Berg, Tinetti, Katz e Lawton \& Brody.

\begin{tabular}{lll}
\hline Escalas & N & \% \\
\hline WOMAC Total & 2 & 12,5 \\
Nenhuma & 6 & 37,5 \\
Pouca & 5 & 31,3 \\
Moderada & 3 & 18,8 \\
Intensa & & \\
Classificação da escala de Berg & 4 & 25,0 \\
Equilíbrio aceitável & 12 & 75,0 \\
Bom equilíbrio & & \\
Risco de queda de Tinetti & 4 & 25,0 \\
Sim & 12 & 75,0 \\
Não & & \\
Classificação da escala de Katz & 10 & 62,5 \\
Independência & 6 & 37,5 \\
Dependência parcial & & \\
Classificação da escala de Lawton \& Brody & & 37,5 \\
Dependência parcial & 6 & 62,5 \\
Independência & 10 & \\
\hline
\end{tabular}

Fonte: Autores (2021).

\section{Discussão}

A osteoartrose é determinada como uma patologia de aspecto crônico-degenerativa que acometem a redução da cartilagem articular, gerando dor, edema, rigidez matinal, redução da amplitude de movimento, acometendo principalmente pessoas de meia idade e idade avançada, gerando dificuldades nas atividades diárias e consequentemente repercutindo na qualidade de vida (Rodrigues et al., 2019).

Neste estudo destaca-se que $81,3 \%(n=13)$ dos idosos são do gênero feminino, fato este semelhante aos estudos de Santos et al. (2015) e Rodrigues et al. (2019) que apresentaram percentual de 69,5\% e 80,7\%, respectivamente. Esta prevalência aumentada, talvez seja decorrente das alterações articulares presentes no gênero feminino com o envelhecimento, na qual destaca-se a osteartrose de joelho, pois como relatado na literatura, após a menopausa ocorre o aumento da degeneração articular (Barbosa, 2008).

No estudo de Franco et al. (2020), assim como no estudo de Alves e Bassit (2013), as médias de idade foram de 67 e

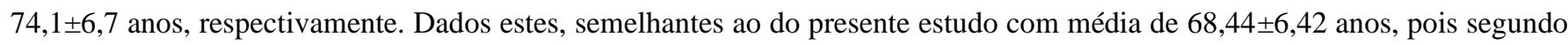
a classificação do envelhecimento pela OMS, as populações destes estudos se encontram na faixa de idoso. Esta idade talvez seja mais prevalente nos estudos, pois ainda é uma faixa etária, na qual os idosos ainda são, na sua maioria, bastante ativos, pois além de realizar as suas atividades diárias, auxiliam nos afazeres domésticos, no cuidado com os netos, entre outros (Cattani \& Perlini, 2004).

Outro aspecto avaliado dos idosos foi o índice de massa corporal (IMC), no qual verificou-se média de 28,97 \pm $3,51 \mathrm{Kg} / \mathrm{cm}^{2}$, que os classifica como sobrepeso. Correlacionando-se com os estudos de Karuka et al. (2011), e Loures et al. (2016), obtiveram IMC de 29,16 $\pm 4,53 \mathrm{~kg} / \mathrm{cm}^{2}$ e $29,3 \pm 4,5 \mathrm{~kg} / \mathrm{cm}^{2}$ em média. Provavelmente, o IMC aumentado é um fator de 
risco para um maior comprometimento funcional, pois altera o centro de gravidade postural do indivíduo, promovendo compensações e desequilíbrios musculares (Loures et al., 2016).

A autonomia e independência podem apresentar algum tipo de declínio com o avançar da idade, isto gera a necessidade de morar com algum parente ou cuidador (Ferreira et al., 2010). Fato este, que pode ser observado no presente estudo, em que $87,5 \%(n=14)$ idosos residem com algum familiar e $81,3 \%(n=13)$ residem com duas ou mais pessoas, como também observado por Alves; Bassit (2013), dos quais 87,5\% viviam em casa própria e 82,1\% com uma ou mais pessoas.

Em relação as patologias associadas, foi observado no presente estudo, frequência média de 31,3\% (n=5). Fato este que não se relaciona aos estudos encontrados na literatura. Segundo Pereira, Nogueira e Silva (2015), a principal doença no idoso com osteoartrose foi hipertensão $(46,2 \%)$, já segundo Mendes et al $^{19}, 80 \%$ dos idosos sofrem de patologias associadas ao processo artrósico.

Sabe-se que durante o processo do envelhecimento, a maioria das doenças crônicas degenerativas não transmissíveis vão se instalando de forma lenta e progressiva, principalmente, a hipertensão arterial sistêmica, a diabetes, a osteoporose e a osteoartrose. Estas alterações podem gerar comprometimentos sistêmicos interferindo nas atividades de vida diária do idoso (Leite et al., 2012).

A osteoartrose de joelho apresenta quadro clínico clássico, como a presença de dor, rigidez articular e diminuição da capacidade física de executar funções do dia a dia, diante disto foi elaborada uma escala específica para esta avaliação clínica do indivíduo, o WOMAC (Vasconcelos, et al., 2008).

Segundo Medeiros et al. (2008), obteve em seu estudo que em média a intensidade de dor foi de 4,6 $\pm 3,8$, a rigidez foi de 1,4 $\pm 1,6$ e o de atividade física 16,1 $\pm 15,0$ relatando baixo nível de comprometimento funcional. Dados estes que não correlacionam ao atual estudo, no qual verificou-se que o escore de intensidade de dor foi, em média, de 25,00 $\pm 23,52$, o de rigidez foi de 29,69 $\pm 26,95$ e o de atividade física, foi de 30,15 $\pm 23,69$, apresentando um nível de comprometimento funcional moderado. Porém ambos os estudos citados, diferem do estudo de Alves e Bassit (2013), que obteve a intensidade de dor, em média, de 53,6 $\pm 20,4$, rigidez foi de 52,2 $\pm 22,4$ e o de atividade física $53,6 \pm 20,3$, apresentando um pior nível de comprometimento funcional, dos estudos acima.

Estas alterações no grau de comprometimento da osteoartrose na funcionalidade dos idosos podem ser decorrentes de alguns fatores, tais como tempo e gravidade da osteoartrose, nível de atividade física, idade, sexo, pois estes fatores podem influenciar diretamente nas repercussões que estes indivíduos apresentam (Duarte et al., 2013).

Além das alterações articulares promovidas pela osteoartrose, é possível que com o desenvolvimento progressivo das limitações, outras alterações sejam observadas, tais como comprometimento no equilíbrio e na marcha, e consequentemente o risco de quedas e lesões associadas a esta (Duarte et al., 2013).

Para avaliação do equilíbrio, a escala de Berg é um instrumento bastante utilizado e disseminado entre pesquisadores. Nos estudos de Antunes et al. (2020), Costa; Carvalho (2012), assim como o presente estudo, averiguou-se bom equilíbrio nas

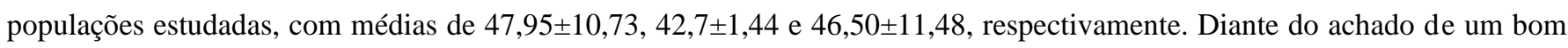
equilíbrio das populações nos estudos apresentados, mostra que apesar dos idosos apresentarem a osteoartrose, não gerou comprometimento nos seus ajustes posturais de equilíbrio, diminuindo assim a possibilidade de quedas. (Marx et al., 2006).

No teste de Tinetti, verificou-se que a população do presente estudo apresentou risco moderado de quedas $(20,38 \pm$ 6,33), dados semelhantes ao estudo de Antunes et al. (2020), e Nogueira et al (2017), que obteveram média de 21,79 $\pm 4,74$ e de 21,12 $\pm 6,18$ pontos. Desse modo, o que indica que os estudos expostos mostram risco moderado de quedas ao avaliar o equilíbrio e a marcha (Karuka et al., 2011).

Para uma avaliação mais fidedigna, sobre a capacidade de realizar as Atividades Básicas de Vida Diária (ABVDs) foi utilizada a escala de Katz, que constatou que a maioria dos idosos foram considerados independentes $(62,5 \%)$. Dados estes 
também encontrados por Santos et al. (2012) e Sudré et al. (2012), que utilizando a mesma escala, obtiveram respectivamente que $72 \%$ e $61,4 \%$ dos idosos entrevistados eram totalmente independentes. Portanto, os estudos acima citados, observaram que os idosos mostram uma boa capacidade funcional e autonomia para gerenciar sua vida em suas atividades da vida diária (Ferreira et al., 2010).

Para avaliar a capacidade de realizar as Atividades Instrumentais de Vida Diária (AIVDs) está a Escala de Lawton e Brody, um questionário bastante usado para a avaliação do idoso. No presente estudo obteve em média de 19,63 $\pm 2,58$ classificando-se como dependência moderada. Dados estes que diferem dos achados de Nogueira; Frota (2015) e Diniz et al. (2018), nas quais os idosos se apresentaram como independentes com médias de $22,34 \pm 4,77$ e $24,87 \pm 5,35$. Talvez a diferença entre os estudos seja decorrente do nível de atividade física em cada população idosa, pois como afirma Novais et al. (2018), quanto mais ativo for o idoso, menores serão suas limitações, melhorando a independência no desempenho das atividades do dia-a-dia.

O presente estudo apresentou algumas limitações devido inclusive a forma que foram respondidos os questionários, pois como foi em ambiente virtual, sem nenhuma interação pesquisador-pesquisado pode ter havido algumas falhas nos preenchimentos das escalas. Também, ao finalizar a organização dos dados, foi observado dados faltantes para complementar os resultados, tais como: nível de atividade física, há quantos anos apresenta a osteoartrose e nível de dor por escala específica para dor.

\section{Considerações Finais}

Neste estudo, a população de idosos com osteoartrose de joelhos avaliada apresentou um nível de comprometimento funcional moderado, porém com equilíbrio preservado, baixo risco a quedas e com independência funcional preservada tanto para as ABVDs e AIVDs.

A aplicação das escalas apresenta-se como importante ferramenta terapêutica para averiguar o nível de alterações decorrentes da osteoartrose associada ao envelhecimento, influenciando na escolha e diferenciação da intervenção fisioterapêutica a ser preconizada para estes indivíduos.

Sugere-se novos estudos, de forma experimental, a fim de buscar informações mais detalhadas e com respaldo científico sobre a utilização dos recursos terapêuticos que auxiliem no tratamento da osteoartrose, na melhora do equilíbrio, da coordenação e na prevenção de quedas em idosos.

\section{Referências}

Alfieri, F. M., Werner, A., Roschel, A. B., Melo, F. C., \& da Silva Santos, K. I. (2009). Mobilidade funcional de idosos ativos e sedentários versus adultos sedentários. Brazilian Journal of Biomotricity, 3(1), 89-94.

Alves, J. C., \& Bassitt, D. P. (2013). Qualidade de vida e capacidade funcional de idosas com osteoartrite de joelho. Einstein (São Paulo), 11, $209-215$.

Antunes, T. B., da Silveira, T. M. V., Barbosa, A. T. V., de Santana, E. J., Gomes, V. M. D. S. A., de Carvalho, V. C. P., \& Uchôa, É. P. B. L. (2020). Avaliação da marcha e do equilíbrio de pacientes idosos com osteoartrose de joelho. Brazilian Journal of Development, 6(9), 72788-72800.

Araújo, F., Pais Ribeiro, J., Oliveira, A., Pinto, C., \& Martins, T. (2008). Validação da escala de Lawton e Brody numa amostra de idosos não institucionalizados. In Actas do $7^{\circ}$ congresso nacional de psicologia da saúde (pp. 655-659). Universidade do Porto.

Araújo, J. G., \& Mejia, D. P. M (2020). A Fisioterapia na artrose de joelho em pacientes da terceira idade: Uma revisão de literatura. Pós-graduação em traumato-ortopedia com ênfase em terapia manual, Faculdade Ávila.

Barbosa, S. R. M. (2008). Identificação de fatores de risco para quedas em idosos, distintos por gênero e idade. [Tese]. Uberlândia: Faculdade de Engenharia Elétrica, Universidade Federal de Uberlândia.

Cassetari, M. R. (2008). Osteoartrose em joelhos como fator limitante para a qualidade de vida em idosos [dissertação]. Botucatu: Universidade Estadual Paulista (UNESP), Faculdade de Medicina de Botucatu. 
Cattani, R. B., \& Girardon-Perlini, N. M. O. (2004). Cuidar do idoso doente no domicílio na voz de cuidadores familiares. Revista eletrônica de enfermagem, $6(2)$.

De Araújo Costa, R. P., \& de Carvalho, M. E. I. M. (2012). Equilíbrio do idoso com osteoartrose de joelho e assintomático. Fisioterapia Brasil, 13(3), 200204.

Dos Santos Schneider, A. R. (2010). Envelhecimento e quedas: a fisioterapia na promoção e atenção à saúde do idoso. Revista Brasileira de Ciências do Envelhecimento Humano, 7(2).

Duarte, V. D. S., Santos, M. L. D., Rodrigues, K. D. A., Ramires, J. B., Arêas, G. P. T., \& Borges, G. F. (2013). Exercícios físicos e osteoartrose: uma revisão sistemática. Fisioterapia em Movimento, 26, 193-202.

Dutra, R. K. D., Belchior. A. C. S., Araújo, F. L. C., Daltro, M. C. S., Costa, M. L. A., Torres, R. B., Garcia, V. V. C. G. (2018). Avaliação da funcionalidade em idosos. Suplemento 2: Faculdades Integradas de Patos, 19, 91-96.

Ferreira, O. G. L., Maciel, S. C., Silva, A. O., Santos, W. S. D., \& Moreira, M. A. S. P. (2010). O envelhecimento ativo sob o olhar de idosos funcionalmente independentes. Revista da Escola de Enfermagem da USP, 44, 1065-1069.

Karuka, A. H., Silva, J. A., \& Navega, M. T. (2011). Análise da concordância entre instrumentos de avaliação do equilíbrio corporal em idosos. Brazilian Journal of Physical Therapy, 15, 460-466.

Leite, L. E. D. A., Resende, T. D. L., Nogueira, G. M., Cruz, I. B. M. D., Schneider, R. H., \& Gottlieb, M. G. V. (2012). Envelhecimento, estresse oxidativo e sarcopenia: uma abordagem sistêmica. Revista Brasileira de Geriatria e Gerontologia, 15, 365-380.

Lino, V. T. S., Pereira, S. R. M., Camacho, L. A. B., Ribeiro Filho, S. T., \& Buksman, S. (2008). Adaptação transcultural da escala de independência em atividades da vida diária (Escala de Katz). Cadernos de saúde pública, 24, 103-112.

Livro de Atas (eBook) do I Congresso de Cuidados Continuados: Dilemas Atuais e Desafios Futuros (2012). (Vol. 1, pp. 178-185). Instituto Politécnico de Bragança, Escola Superior de Saude.

Loures, F. B., Góes, R. F. D. A., Labronici, P. J., Barretto, J. M., \& Olej, B. (2016). Avaliação do índice de massa corporal como fator prognóstico na osteoartrose do joelho r. Revista Brasileira de Ortopedia, 51, 400-404.

Marx, F. C., Oliveira, L. M. D., Bellini, C. G., \& Ribeiro, M. C. C. (2006). Tradução e validação cultural do questionário algofuncional de Lequesne para osteoartrite de joelhos e quadris para a língua portuguesa. Revista Brasileira de Reumatologia, 46, 253-260.

Medeiros, M. M. D. C., Sousa, D. C., Paiva, G. A., Figueiredo, L. M., Freitas, T. H., Soares, D. C., \& Cô, C. M. P. (2008). A screening tool for knee pain (KNEST) in primary care: translation, cultural adaptation and results of the application among Brazilian elderly people living in the community. Geriatrics, Gerontology and Aging, 2(4), 144-150.

Nogueira, L. V., de Oliveira, M., Van Der Haagen, M., Santos, R. D. C. C. S., \& de Seixas Rodrigues, E. L. (2017). Risco de quedas e capacidade funcional em idosos. Revista da Sociedade Brasileira de Clínica Médica, 15(2), 90-93.

Nogueira, P. S. F. (2015). Análise da capacidade funcional de idosos com hanseníase através de três instrumentos. [Tese]. Fortaleza: Faculdade de Farmácia, Odontologia e Enfermagem, Universidade Federal do Ceará.

Pereira, D. S., Nogueira, J. A. D., \& Silva, C. A. B. D. (2015). Qualidade de vida e situação de saúde de idosos: um estudo de base populacional no Sertão Central do Ceará. Revista brasileira de geriatria e gerontologia, 18, 893-908.

Pinheiro, S. C. B., Barrena, H. C., \& Macedo, A. B. (2019). Alterações articulares causadas pelo envelhecimento e seus impactos para a autonomia do idoso. Arquivos do MUDI, 23(3), 35-45.

Rodrigues, R. E., Duarte, P. H. M., \& Feitosa, C. Â. L. (2019). Impacto da osteoartrose de joelho na capacidade funcional e qualidade de vida de pacientes atendidos em um município de Pernambuco, Brasil. Archives Of Health Investigation, 8(7).

Santos, J. P. M., Andraus, R. A., Pires-Oliveira, D. A., Fernandes, M. T., Frâncica, M. C., Poli-Frederico, R. C., \& Fernandes, K. B. (2015). Análise da funcionalidade de idosos com osteoartrite. Fisioterapia e Pesquisa, 22, 161-168.

Santos, N. G. B., Neto, E. M. F., Arêas, G. P. T., da Silva Arêas, F. Z., Leite, H. R., Ferreira, M. A. C., \& Júnior, R. C. F. (2012). Capacidade funcional e qualidade de vida em idosos com osteoartrose no município de Coari-AM. Revista Pesquisa em Fisioterapia, 2(2).

Santos, N. G. B., Neto, E. M. F., Arêas, G. P. T., da Silva Arêas, F. Z., Leite, H. R., Ferreira, M. A. C., \& Júnior, R. C. F. (2012). Capacidade funcional e qualidade de vida em idosos com osteoartrose no município de Coari-AM. Revista Pesquisa em Fisioterapia, 2(2).

Silva, J. M. N. D., Barbosa, M. F. D. S., Castro, P. D. O. C. N. D., \& Noronha, M. M. (2013). Correlação entre o risco de queda e au tonomia funcional em idosos institucionalizados. Revista Brasileira de Geriatria e Gerontologia, 16, 337-346.

Sudré, M. R. S., Reiners, A. A. O., Nakagawa, J. T. T., Azevedo, R. C. D. S., Floriano, L. A., \& Morita, L. H. M. (2012). Prevalência de dependência em idosos e fatores de risco associados. Acta Paulista de Enfermagem, 25, 947-953. 